\title{
HIGHLIGHT
}

\section{Pygmy mouse gene mutation protects against obesity}

\author{
J P Berg \\ Hormone and Central Laboratory, Aker University Hospital, Trondheimsveien 235, 0514 Oslo, Norway
}

(Correspondence should be addressed to J P Berg; Email: j.p.berg@ioks.uio.no)

Of the four spontaneous mouse mutations resulting in impaired growth, the pygmy mouse is extraordinary because it has no aberrations in pathways involving growth hormone or insulin-like growth factors. The pygmy phenotype is caused by a defect in the HMGI-C gene, which is a member of the HMGI family of proteins $(1,2)$.

HMGI proteins belong to a general class of high mobility group (HMG) DNA binding proteins (reviewed in (3)). These are ubiquitous and abundant non-histone nuclear proteins and are divided into three subfamilies. The HMG-1/-2 subfamily has the HMG-1 box as its major functional motif, which consists of approximately 80 amino acids. The box binds DNA to the minor groove and induces specific DNA deformations. A nucleosomal binding domain is the functional motif in the HMG-14/ -17 subfamily. Two molecules of HMG-14 or HMG-17 bind to the nucleosome core, which is the building block of the chromatin fibre, and change the chromatin structure. The HMGI group is the third subfamily and consists of HMG-I and HMG-Y, which are encoded by the same gene and made by alternative splicing, and HMGI-C. The functional domain of these proteins is the AT hook, which is made of nine positively charged amino acids. They bind to the minor groove of DNA with relatively low specificity with regard to the DNA sequence, and preferentially to the sequence $\mathrm{AA}(\mathrm{T} / \mathrm{A}) \mathrm{T}$. The proteins are a part of the structural elements of metaphase chromosomes. HMGI proteins may attenuate the inhibitory effect of the nuclear protein histone $\mathrm{H} 1$ on transcription by displacing $\mathrm{H} 1$ from DNA. They can also affect embryonic development by interfering with homeodomain binding proteins. In addition, the HMGI proteins can modulate the transcription of several genes. They may enhance or repress transcription by direct DNA interactions or by binding to specific transcription factors. Stimulatory effects of HMG-I/Y on transcription have been studied in detail on the virus-inducible enhancer of the interferon- $\beta$ gene. Binding of HMG proteins stabilises DNA and specific transcription factors in a higher order nucleoprotein complex called an enhanceosome (reviewed in (4)).

The expression of HMGI-C has been studied in detail in mouse embryos (5). At an early embryonic stage HMGI-C mRNA is found in all tissues, whereas the pattern of expression is restricted particularly to mesenchymal derivatives, some epithelial cell layers, and parts of the central nervous system in the second half of embryonic development.

HMGI-C expression is normally not detected in adult tissues from humans or mice $(5,6)$. However, HMGI-C mRNA is frequently found in solid tumours in humans such as lipomas, pleomorphic adenomas of the salivary gland, uterine leiomyomas, and lung hamartomas. It has been shown that these tumours often have chromosome 12 rearrangements, which directly affect the HMGI-C gene on this chromosome (7). Expression of HMGI-C seems to be linked to proliferation both in normal embryos and in tumour cells.

In a recent paper, Anand \& Chada (8) showed that the expression of HMGI-C in fat depots from normal mice was detectable after 1 week of a high-fat diet. Expression of the gene was also observed in fat from the genetically obese mouse strains $L e p^{o b} / L^{o p}{ }^{o b}$ and $L e p r^{d b} /$ Lepr $^{d b}$, which lack leptin or its receptor, respectively. Adipose tissue from normal mice on a standard diet did not express the gene. The data indicated a role for HMGI-C in obesity. This was further elaborated by feeding a strain of pygmy mouse carrying two non-functional HMGI-C alleles $\left(\mathrm{Hmgic}^{-/-}\right)$, heterozygotes $\left(\mathrm{Hmgic}^{+-}\right)$, and wild-type mice with standard or high-fat diets. Interestingly, both the $\mathrm{Hmgic}^{-/-}$and the Hmgic ${ }^{+/-}$mice did not increase in weight on a high-fat diet compared with the controls on a standard diet, whereas the wild-type mice increased in weight compared with their controls as expected. On a standard diet, the weight of the heterozygotes was not significantly different from the wild-type mice. There were no differences in food intake. The observations indicated that lack of one or both of the HMGI-C alleles may protect against obesity induced by a high-fat diet and there seemed to be a haploinsufficiency effect of HMGI-C.

Anand \& Chada (8) also studied effects of HMGI-C in the genetically obese $L e p^{o b} / L e p^{o b}$ mice, which lack a functional leptin gene, by knocking out their HMGI-C gene also. Lack of HMGI-C resulted in a remarkable resistance against obesity, and the weights of the mice were only $27 \%$ of those of the leptin-deficient controls by 30 weeks of age. In comparison, the weights of the $\mathrm{Hmgic}^{-/-}$mice with a normal leptin gene were $47 \%$ of those of the wild-type controls. Leptin-deficient mice, which were heterozygous for the HMGI-C null mutation, had reduced expression of HMGI-C and their weights were $72 \%$ of those of the HMGI-C heterozygotes 
with a normal leptin gene. Greatest weight reduction was observed in the mesenteric fat pads. Both the appearance of the adipocytes and the expression of genes involved in adipogenesis were similar in leptindeficient mice with or without functional HMGI-C. The decrease in fat-pad weight was therefore most likely to have been caused by a reduction in cell number. Although the absence of functional HMGI-C attenuated the weight increase in leptin-deficient mice, they were still heavier than wild-type mice, indicating that there are pathways for adipocyte proliferation independent of HMGI-C.

Absence of HMGI-C reduced the accumulation of fat normally observed in the leptin-deficient mice, but other phenotypic characteristics of leptin deficiency were still present. As evaluated by the concentrations of plasma insulin and glucose, and glucosuria, the relatively lean double-homozygous HMGI-C and leptin-deficient mice were as insulin resistant as their obese leptin-deficient relatives with a normal HMGI-C gene. The study by Anand \& Chada (8) indicates that insulin resistance in the $L e p^{o b} / L e p^{o b}$ mouse is primarily caused by leptin deficiency and not by obesity, and indirectly supports the findings of previous studies in which anti-diabetic effects of leptin have been demonstrated ((9); reviewed in (10)).

HMGI-C facilitates the interaction between transcription factors and DNA, and seems to determine the growth potential of pre-adipocytes. The absence of HMGI-C expression in normal adult tissues, the appearance of HMGI-C in adipose tissue in obesity, and the gene-dosage effect observed in the studies of Anand \& Chada (8) makes HMGI-C an ideal peripheral target for the treatment of obesity and a complement to interventions aimed at appetite suppression.

\section{References}

1 Benson KF \& Chada K. Mini-mouse: phenotypic characterization of a transgenic insertional mutant allelic to pygmy. Genetic Research 199464 27-33.

2 Zhou X, Benson KF, Ashar HR \& Chada K. Mutation responsible for the mouse pygmy phenotype in the developmentally regulated factor HMGI-C. Nature 1995376 771-774.

3 Bustin M. Regulation of DNA-dependent activities by the functional motifs of the high-mobility-group chromosomal proteins. Molecular and Cellular Biology 199919 5237-5246.

4 Carey M. The enhanceosome and transcriptional synergy. Cell $1998925-8$.

5 Hirning-Folz U, Wilda M, Rippe V, Bullerdiek J \& Hameister H. The expression pattern of the Hmgic gene during development. Genes, Chromosomes and Cancer 199823 350-357.

6 Rogalla P, Drechsler K, Frey G, Hennig Y, Helmke B, Bonk U \& Bullerdiek J. HMGI-C expression patterns in human tissues. Implications for the genesis of frequent mesenchymal tumors. American Journal of Pathology 1996149 775-779.

7 Schoenmakers EF, Wanschura S, Mols R, Bullerdiek J, Van den Berghe H \& Van de Ven WJ. Recurrent rearrangements in the high mobility group protein gene, HMGI-C, in benign mesenchymal tumours. Nature Genetics $199510436-444$.

8 Anand A \& Chada K. In vivo modulation of Hmgic reduces obesity. Nature Genetics 200024 377-380.

9 Shimomura I, Hammer RE, Ikemoto S, Brown MS \& Goldstein JL. Leptin reverses insulin resistance and diabetes mellitus in mice with congenital lipodystrophy. Nature 1999401 73-76.

10 Berg J. Leptin is a potent anti-diabetic in mice with lipodystrophy and insulin resistance. European Journal of Endocrinology 2000 $142114-116$ 